\title{
Significance of biological membranes for accurate computational dosimetry of low frequency electric fields
}

\author{
S. Marzban ${ }^{1}$, M. Saviz ${ }^{1}$, F. Towhidkhah ${ }^{1}$ \\ 1. Biomedical Engineering Department, Amirkabir University of Technology, Tehran, Iran \\ 2. E-mail any correspondence to: msaviz@aut.ac.ir
}

\begin{abstract}
Computational dosimetry has become the main tool for estimating induced electric fields within brain tissues in transcranial direct current stimulation (tDCS) which is recently attracting the attention of researches for motor function disturbances such as Parkinson's disease. This paper investigates the effect of including or excluding the very thin meninges in computing tDCS electric fields using CST software. For this purpose, two models of the brain with and without meninges were used to induce electric field with two DC current electrodes $(2 \mathrm{~mA})$ in regions of the model referring to $\mathrm{M} 1$ and Prefrontal Cortex $\left(\mathrm{FP}_{2}\right)$ similar to tDCS. Considering meninges, the results have shown differences in the induced field showing that there might be problems with conventional models in which meninges are not taken into account.
\end{abstract}

Keywords: tDCS, modeling, electric field, meninges

\section{Introduction}

Transcranial electrical stimulation using weak current may be a promising tool to modulate cerebral activity in a noninvasive, and to some extent reversible, selective way [1]. Transcranial direct current stimulation (tDCS) is a useful noninvasive technique that imposes a weak direct electrical current (1-2 $\mathrm{mA}$ ) to modulate the activity of neurons in the brain. The experiments suggested that electrodes placed on the head can produce noticeable neurological changes depending on the current direction [2]. The current results in an electric field, and to attain this field in specific regions of the brain, the electric current should pass through different head layers via skin, fat, skull, meninges, and cortex (part of the brain). In order to model the brain, different layers should be considered, including gray and white matters.

The meninges, three layers of protective tissue, cover the outer surface of the central nervous system (brain and spinal cord) and comprise three connective tissue layers viz. (from the innermost to the outermost layer) the pia mater, arachnoid and the dura mater. The meninges also support blood vessels and contain cerebrospinal fluid in between. The pia mater is in direct contact with the cortex, and there is space for the highly conductive cerebrospinal fluid (CSF) between the pia mater and the arachnoid [3]. The dura mater is a thin layer that has low conductivity closest to the skull and vertebrae. Figure 1 shows the layers of the meninges in the brain [4].

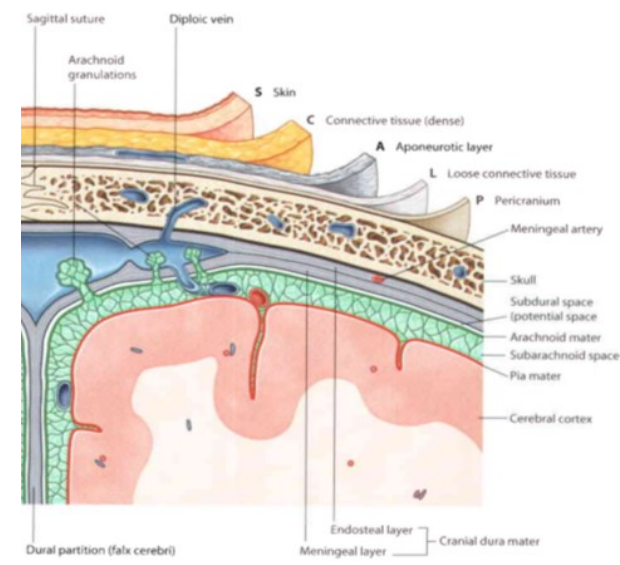

Fig. 1: Cross-section of meninges in brain [4]. 
In most voxel-based computational models of human brain, the resolution is on the order of millimeters, and fine structures like the meninges are ignored. This leads to negligible error in high frequency simulations, since the capacitive barrier of thin, low conductivity layers tend to be shorted out at high frequencies. In contrast, high discrepancies are expected at lower frequencies. This paper addresses the estimation of amplitude $E$, electric field due to DC current applied, and J, current density due to the tissue conductivity, by inducing electric current from M1 to $\mathrm{FP}_{2}$ in two models of the head while considering and ignoring meninges. It also compares these two models in terms of electric field distribution, in order to estimate the computational errors introduced by ignoring the thin layers.

The dosimetric significance of low conductivity biological membranes has been pointed out in a different context by Miller [5] who demonstrates that the vitelline membrane can electrically isolate the yolk from the rest of the egg. Therefore, the homogeneous approximation is insufficient for detailed dosimetric considerations. Parazini et al. [6] have recently modeled the brain and induced electric field in a realistic human head model, but without considering the meninges. Moreover, Chang-Hwan Im et al. [7] evaluated the local electric fields which is generated by tDCS but they ignored meninges.

\section{Materials and methods}

A commercial software package (CST EM STUDIO ${ }^{\circledR}$ (CST EMS)) was used to design electrodes and to model a layered human head model. The circular electrodes were designed with electric properties of perfect conductors of $0.01 \mathrm{~cm}$ thickness and $7.068 \mathrm{~cm}^{2}$ area, situated at sites commonly used in the tDCS on $\mathrm{M} 1$ and $\mathrm{FP}_{2}$. Figures 2 and 3 show the geometry of the electrodes, the current direction, and the model layers, respectively. The real head model consists of different layers.

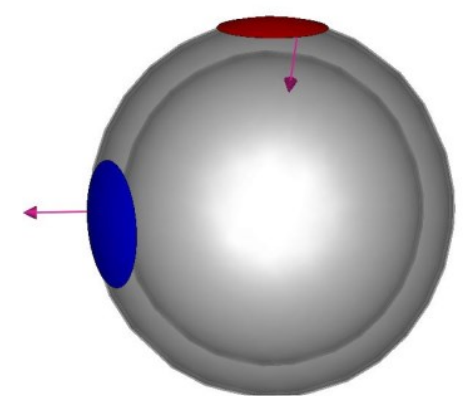

Fig. 2: The geometry of electrode and the current direction.

In this research, two different models were simulated and proposed as follows:

- The conventional model with 4 layers, viz. skin, fat, skull, and the grey matter of cortex in which the injected current was simulated with $2 \mathrm{~mA}$ amplitude and the whole model consisted of a mesh grid of 2437355 tetrahedrons. This simulation needed 1427 seconds run-time.

- The full model with 7 layers, viz. skin, fat, skull, dura mater, arachnoid mater that includes CSF (cerebrospinal fluid), pia mater, and the grey matter in which the injected current was the same as the former model and a mesh grid of 2572507 tetrahedrons. This simulation took 1627 seconds.

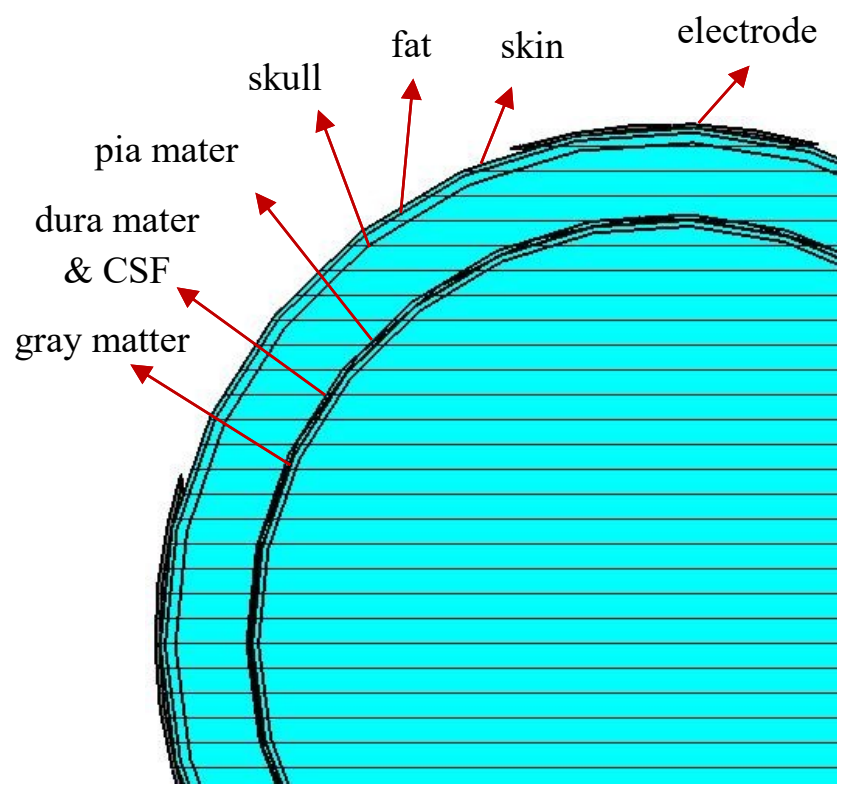

Fig. 3: The geometry of layers.

Conductivity of each brain tissue at low frequencies [8] with thickness of each layer is shown in Table 1 . There are two equations in electrostatics that are solved together to provide the final field values in a stationary current solver:

$$
\begin{aligned}
& \vec{\nabla} \cdot \vec{J}=0 \\
& \vec{J}=\sigma \vec{E}
\end{aligned}
$$

Where $\mathrm{E}$ is the electric field, $\sigma$ the conductivity, and $\mathrm{J}$ is the current density. Furthermore the tangential E field component was continuous at the interface between two materials $\left(\mathrm{E}_{1 t}=\mathrm{E}_{2 t}\right.$, which results in $\left.\frac{J_{1}}{\sigma_{1}}=\frac{J_{2}}{\sigma_{2}}\right)$.

After stimulation with $2 \mathrm{~mA} \mathrm{DC}$ current, depending on the conductivity of the material, the electric field is computed in the model. The amplitude distribution of electric field in the two head models were computed and analyzed. Furthermore, the electric field was computed in bisector of two electrodes from the center of head model to the skin, the external layer, to observe the differences.

\section{Ethical approval}

The conducted research is not related to either human or animal use. 
Table 1: Brain tissue dielectric properties

\begin{tabular}{lcc}
\hline Brain layer & Thickness & Conductivity $(\mathrm{S} / \mathrm{m})$ \\
\cline { 2 - 3 } (a) & $0.5 \mathrm{~mm}$ & 0.001 \\
Fat & $1 \mathrm{~mm}$ & 0.05 \\
Skull & $7 \mathrm{~mm}$ & 0.05 \\
Dura mater & $0.5 \mathrm{~mm}$ & 0.0025 \\
$\begin{array}{l}\text { Arachnoid } \\
\text { mater and CSF }\end{array}$ & $40 \mu \mathrm{m}$ & 2 \\
$\begin{array}{l}\text { Pia mater } \\
\text { Grey matter }\end{array}$ & $0.6 \mathrm{~mm}$ & 0.05 \\
\hline
\end{tabular}

(b)

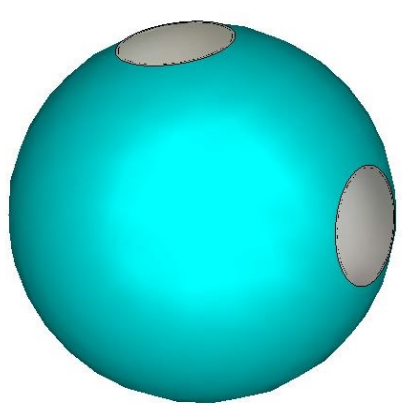

(c)
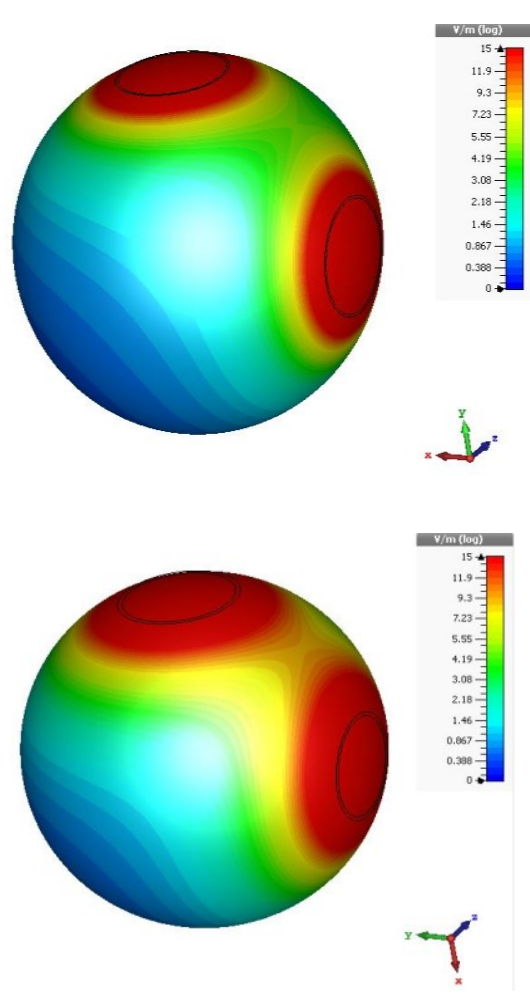

Fig. 4: Geometry of electrodes on human head (a), and electric field distribution in the head model without and with considering meninges while injected current equal to $2 \mathrm{~mA}$ ( $b$ and $c$ ).

Results

Figure 4 shows the geometry of electrodes and where they are situated on human head (a), and the amplitude distribution of electric field in the head model without and with considering meninges ( $b$ and $c$ ). The difference of colors shows a meaningful difference in the distribution of the electric field.

As shown in the figure, the distribution of color ranges from blue to red, i.e. 0 to $15 \mathrm{~V} / \mathrm{m}$. The highest intensity is related to red and the lowest to the blue through the color spectrum. Different results of amplitude distribution of electric field in the head model is obviously seen in the figure.

It is obvious that surface electric field intensity in figure 4 (c) is higher than that in figure 4 (b). This is caused by low conductivity in the layers of meninges.

Figure 5 shows the electric field in the bisector of two electrodes in both head models, from the center of the model to the external layer. The electric field from the center of the anode electrode to the center of the head model in both models are demonstrated. In this figure the difference is clearly obvious. Moreover in figure 5 and 6 the hypothetical line from the center of head model to the skin and from the center of the anode electrode through the center of the head model is demonstrated.

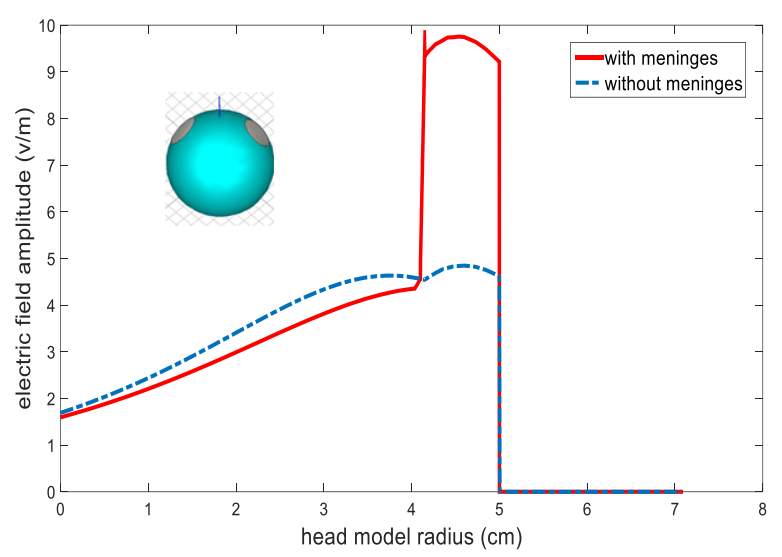

Fig. 5: Electric field in the hypothetical line in the bisector of the electrodes from the center to the skin in the two models.

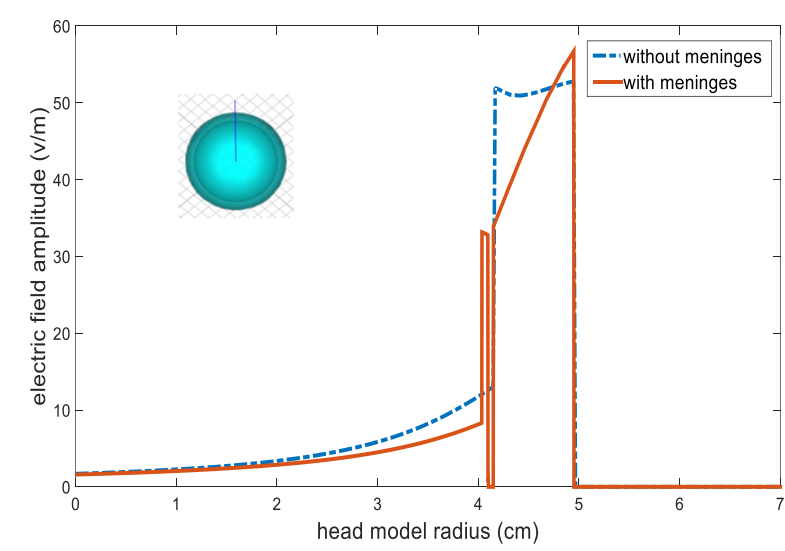

Fig. 6: Electric field in the hypothetical line from the center of the head model to the center of the anode electrode. 
As shown in figure 5, the diagrams depict variation of the electric field from the center to the external layer in the model with and without considering meninges.

\section{Discussion}

It can be concluded that modeling the tDCS without taking meninges into account can lead to an error of about $15 \%$ in evaluating fields within the cortex and gray matter between electrodes. This obviously calls for including the biological membranes in computational models, especially for DC or low frequency simulations.

\section{Acknowledgement}

Special thanks go to Mr. Farhad Alizadeh, M.Sc. student of Amirkabir University, for his helpful information regarding the software usage, and to Mrs. Zhila Ghaemi, faculty at Asrar Institute of Higher Education, for her valuable comments on English writing issues of the manuscript.

\section{Conflict of interest}

Authors state no conflict of interest.

\section{References}

1. M. A. Nitsche and W. Paulus, "Excitability changes induced in the human motor cortex by weak transcranial direct current stimulation," The Journal of Physiology, vol. 527, pp. 633-639, 2000.

https://doi.org/10.1111/j.1469-7793.2000.t01-1-00633.x
2. P. Auvichayapat and N. Auvichayapat, "Basic knowledge of transcranial direct current stimulation," Journal of the Medical Association of Thailand, vol. 94, p. 518, 2011.

3. S. Grimnes and O.G. Martinsen, Bioelectricity and Bioimpedance Basics. $2^{\text {nd }}$ ed. London: Academic, 2008.

4. R. Drake, A. W. Vogl, and A. W. M. Mitchell, Dorland's/Gray's Pocket Atlas of Anatomy E-Book: Elsevier Health Sciences, 2008.

5. D. L. Miller, "Electric fields induced in chicken eggs by $60-\mathrm{Hz}$ magnetic fields and the dosimetric importance of biological membranes," Bioelectromagnetics, vol. 12, pp. 349-360, 1991. https://doi.org/10.1002/bem.2250120604

6. M. Parazzini, S. Fiocchi, E. Rossi, A. Paglialonga, and P. Ravazzani, "Transcranial direct current stimulation: estimation of the electric field and of the current density in an anatomical human head model," IEEE Transactions on Biomedical Engineering, vol. 58, pp. 1773-1780, 2011. https://doi.org/10.1109/TBME.2011.2116019

7. C.-H. Im, J.-H. Park, M. Shim, W. H. Chang, and Y.-H. Kim, "Evaluation of local electric fields generated by transcranial direct current stimulation with an extracephalic reference electrode based on realistic 3D body modeling," Physics in Medicine and Biology, vol. 57, p. 2137, 2012. https://doi.org/10.1088/0031-9155/57/8/2137

8. S. Gabriel, R. Lau, and C. Gabriel, "The dielectric properties of biological tissues: III. Parametric models for the dielectric spectrum of tissues," Physics in Medicine and Biology, vol. 41, p. 2271,1996 . https://doi.org/10.1088/0031-9155/41/11/003 